\title{
clAP2 is a ubiquitin protein ligase for BCL10 and is dysregulated in mucosa-associated lymphoid tissue lymphomas
}

\author{
Shimin Hu, ${ }^{1}$ Ming-Qing Du,,2 Sun-Mi Park, ${ }^{3}$ Allison Alcivar, ${ }^{1}$ Like Qu, ${ }^{1}$ Sanjeev Gupta, ${ }^{4}$ Jun Tang, ${ }^{1}$ \\ Mathijs Baens, ${ }^{5}$ Hongtao Ye, ${ }^{2}$ Tae H. Lee, ${ }^{3}$ Peter Marynen, ${ }^{5}$ James L. Riley, ${ }^{4}$ and Xiaolu Yang ${ }^{1}$ \\ ${ }^{1}$ Abramson Family Cancer Research Institute and Department of Cancer Biology, University of Pennsylvania School of Medicine, \\ Philadelphia, Pennsylvania, USA. ${ }^{2}$ Department of Pathology, Division of Molecular Histopathology, University of Cambridge, Cambridge, United Kingdom. \\ ${ }^{3}$ Department of Biology and Protein Network Research Center, Yonsei University, Seoul, South Korea. ${ }^{4}$ Abramson Family Cancer Research Institute and \\ Department of Pathology and Laboratory Medicine, University of Pennsylvania School of Medicine, Philadelphia, Pennsylvania, USA. \\ 5Human Genome Laboratory, Center for Human Genetics and Flanders Interuniversity Institute for Biotechnology, Leuven, Belgium.
}

\begin{abstract}
The pathogenesis of mucosa-associated lymphoid tissue (MALT) lymphomas is associated with independent chromosomal translocations that lead to the upregulation of either BCL10 or MALT1 or the generation of a fusion protein, cIAP2-MALT1. While both BCL10 and MALT1 are critically involved in antigen receptormediated NF- $\kappa B$ activation, the role of cIAP2 is not clear. Here we show that cIAP2 is a ubiquitin ligase (E3) of BCL10 and targets it for degradation, inhibiting antigen receptor-mediated cytokine production. cIAP2MALT1 lacks E3 activity, and concomitantly, the BCL10 protein is stabilized in MALT lymphomas harboring this fusion. Furthermore, BCL10 and cIAP2-MALT1 synergistically activate NF- $\kappa B$. These results reveal cIAP2 as an inhibitor of antigenic signaling and implicate its dysfunction in MALT lymphomas.
\end{abstract}

\section{Introduction}

Inhibitor of apoptosis proteins (IAPs) are a family of evolutionarily conserved proteins that regulate apoptosis and other cellular processes $(1,2)$. IAPs contain 1-3 characteristic baculoviral IAP repeat (BIR) motifs, which can directly bind to and inhibit caspases, the central executioner proteases of apoptosis (3). This inhibitory effect of IAPs on caspases is counteracted during apoptosis by death inducers such as mammalian Smac/DIABLO (4-6). However, some IAPs, including cellular IAP1 (cIAP1) and cIAP2, are poor inhibitors of caspases, and they may have important functions in addition to or instead of inhibiting apoptosis. cIAPs and several other IAPs also contain a RING domain, which possesses ubiquitin ligase (E3) activity that may mediate the degradation of themselves and proapoptotic proteins such as Smac/DIABLO (7-9). cIAP1 targets the antiapoptotic molecule TRAF2 for degradation and thus plays a role in promoting apoptosis (10).

cIAP2 is implicated in the mucosa-associated lymphoid tissue (MALT) lymphomas; this presents a definitive example of the involvement of IAPs in human diseases. MALT lymphomas are the most common type of human lymphomas that arise in extranodal sites. They affect virtually every organ in the human body; the majority occur in the stomach $(11,12)$. The pathogenesis of MALT lymphomas is specifically associated with at least 3 independent chromosomal translocations. Two translocations, $\mathrm{t}(1 ; 14)(\mathrm{q} 22 ; \mathrm{q} 32)$ and $\mathrm{t}(14 ; 18)(\mathrm{q} 32 ; \mathrm{q} 21)$, result in the upregulation of BCL10 (an adaptor protein with a caspase recruitment domain, or CARD) and MALT1 (a paracaspase related to caspases), respectively, because of the juxtaposi-

Nonstandard abbreviations used: BIR, baculoviral IAP repeat; CARD, caspase recruitment domain; cIAP, cellular inhibitor of apoptosis protein; GST, glutathione-Stransferase; IAP, inhibitor of apoptosis protein; MALT, mucosa-associated lymphoid tissue; siRNA, small interfering RNA.

Conflict of interest: The authors have declared that no conflict of interest exists.

Citation for this article: J. Clin. Invest. 116:174-181 (2006)

doi:10.1172/JCI25641. tion of the corresponding genes with the Ig heavy chain locus (13-17). The third and most common translocation, t(11;18)(q21; 21$)$, generates a fusion protein between cIAP2 and MALT1 (18-20). MALT lymphomas with these distinct translocations present no apparent difference in their clinical and pathological features, indicating that there may be a unifying molecular mechanism for the tumors. Indeed, both BCL10 and MALT1 play a critical role in antigen receptor-mediated lymphocyte proliferation and signaling to NF- $\kappa \mathrm{B}$, which controls the expression of genes critical for cell survival, proliferation, and immune responses (21-24). BCL10 and MALT1 function together to promote lysine 63-linked polyubiquitination of NF- $\mathrm{BB}$ essential modifier (NEMO), a regulatory component of the inhibi-

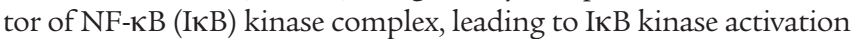

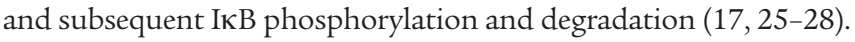
The cIAP2-MALT1 fusion strongly activates NF- $\kappa \mathrm{B}$ compared with MALT1, suggesting that it is a gain-of-function mutant of MALT1 $(17,25,29)$. However, the function of cIAP2 as related to MALT lymphomas is not understood. Specifically, it is unclear whether cIAP2, like BCL10 and MALT1, is involved in antigen receptor signaling and how its translocation may contribute to the pathogenesis of MALT lymphomas. In the current study, we present evidence that cIAP2 is an E3 ubiquitin ligase of BCL10 and targets it for degradation. This function of cIAP2 is lacking in cIAP2-MALT1, and this leads to enhanced stability of BCL10 in $\mathrm{t}(11 ; 18) /$ cIAP2-MALT lymphomas that likely contributes to cIAP2-MALT1-induced NF-кB activation. Thus, all 3 proteins involved in MALT lymphomas are functionally linked in antigen receptor signaling to NF-кB.

\section{Results}

The BCL10 protein is stabilized in t(11;18)/cIAP2-MALT1 lymphomas. The COOH-terminal RING domain of cIAP2 is always deleted in cIAP2MALT1 fusions found in $\mathrm{t}(11 ; 18)$ MALT lymphomas $(18,30)$, leading us to reason that cIAP2 may normally target a protein related to MALT lymphomas for degradation and that this function may be lost 
A

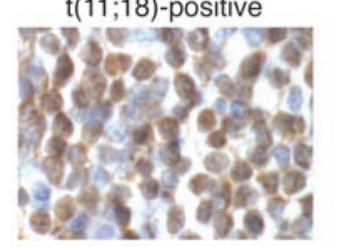

B

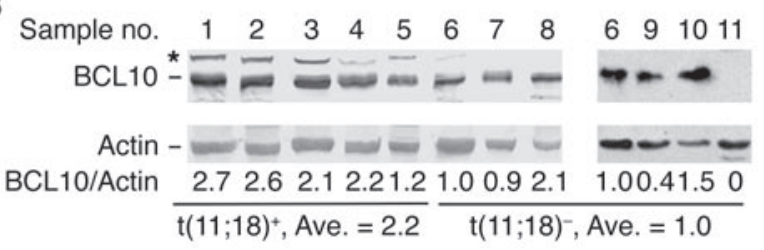

Figure 1

$\mathrm{BCL} 10$ expression level is enhanced in $\mathrm{t}(11 ; 18) / C / A P 2-M A L T 1$ lymphomas. (A) Immunohistochemistry analysis of BCL10 expression in $\mathrm{t}(11 ; 18)$-positive (left) and -negative (right) MALT lymphomas without $\mathrm{t}(1 ; 14) / / g H-B C L 10$ and $\mathrm{t}(14 ; 18) / / g H-M A L T 1$ translocation. Formalinfixed paraffin-embedded tissue sections from MALT lymphomas were immunostained with anti-BCL10 antibody. Original magnification, $\times 400$. (B) Comparison of BCL10 expression levels in $t(11 ; 18)$-positive and -negative MALT lymphomas. Extracts made from MALT lymphomas were analyzed by Western blotting with antibodies against BCL10 (top) and actin (bottom). Samples 1-5: t(11;18)-positive MALT lymphomas; samples $6-11$ : $\mathrm{t}(11 ; 18)$-negative MALT lymphomas with neither $\mathrm{t}(1 ; 14) / / g H-B C L 10$ nor $\mathrm{t}(14 ; 18) / / g H-M A L T 1$. The ratios of $\mathrm{BCL} 10$ to actin signals relative to that of sample 6 and the average (Ave.) ratios among $\mathrm{t}(11 ; 18)$-positive and -negative samples are given. Each sample contained approximately $70 \%$ tumor cells and $30 \%$ T lymphocytes and stromal cells. *Phosphorylated BCL10.

in cIAP2-MALT1. We further reasoned that this cIAP2 target might be BCL10. To explore this possibility, we first determined whether the level of BCL10 protein is enhanced in $\mathrm{t}(11 ; 18) /$ IAP2-MALT1 MALT lymphoma cells. Immunohistochemical analysis showed that the $t(11 ; 18)$-positive tumors expressed significantly higher levels of BCL10 than the $\mathrm{t}(11 ; 18)$-negative ones (Figure 1A), consistent with previous studies and a recent one on a large number of MALT lymphomas (30-32). To quantitate the difference in BCL10 expression in these tumors, we analyzed $5 \mathrm{t}(11 ; 18)$-positive and $6 \mathrm{t}(11 ; 18)$-negative MALT lymphomas by Western blot analysis. All tumor samples were at advanced stages and contained similar levels of tumor cells. On average, BCL10 was expressed more than twice as much in the $\mathrm{t}(11 ; 18)$-positive MALT lymphomas as in the $\mathrm{t}(11 ; 18)$-negative MALT lymphomas (Figure 1B, samples $1-5$ versus $6-11, P<0.0176$ ). These tumor samples contained reactive $T$ lymphocytes and stromal cells (about 30\%), which express significantly lower levels of BCL10 (33). Thus, the actual difference of BCL10 levels in $\mathrm{t}(11 ; 18)$-positive and -negative cells was about 3 -fold, in agreement with the dramatic difference of BCL10 levels detected by immunohistochemistry. In addition, we have previously found no significant difference in BCL10 mRNA expression between MALT lymphomas with and without $\mathrm{t}(11 ; 18)$, including samples used in this study (32). Taken together, these results suggest that the BCL10 protein is stabilized in MALT lymphomas expressing cIAP2-MALT1.

cIAP2 associates with BCL10 through its BIR region. We next examined whether cIAP2 interacts with BCL10. HA-tagged BCL10 was expressed either alone or together with FLAG-tagged cIAP2 in human embryonic kidney (HEK) 293T cells, and the lysates from transfected cells were immunoprecipitated with an anti-
FLAG antibody. BCL10 was readily detected in the anti-FLAG immunoprecipitates from cells coexpressing CIAP2 and BCL10 but not in those from cells expressing BCL10 alone (Figure 2B, lane 2 versus 1). Ectopically expressed cIAP2 also bound to endogenous BCL10 (see below). Additionally, we performed an in vitro binding assay. In vitro-translated and metabolically labeled cIAP2 bound to a recombinant glutathione-S-transferase (GST) fusion of BCL10 but not to GST (Figure 2C, lanes 1-3). To determine whether endogenous BCL10 and CIAP2 associate with each other, extracts from unstimulated and PMA/ionomycin-stimulated human primary $\mathrm{T}$ cells were immunoprecipitated with anti-BCL10 antibody and an isotype-matching control antibody. The immunoprecipitated proteins were analyzed by Western blot. This analysis revealed that endogenous BCL10 and cIAP2 specifically associated with each other in primary lymphocytes, and this interaction was somewhat enhanced when the cells were becoming activated (Figure 2D).

When expressed in mammalian cells, BCL10 appeared to be phosphorylated, based on the appearance of slower migrating bands on SDS-PAGE and the disappearance of these bands upon phosphatase treatment (Figure 2, B and E). The phosphorylation of BCL10 has been reported to correlate with its membrane recruitment and activation (34). Interestingly, the cIAP2:BCL10 interaction appeared to be regulated by BCL10 phosphorylation, as phosphorylated BCL10 bound much more strongly to cIAP2 compared with unphosphorylated BCL10 (Figure 2B, compare the top and bottom panels). To investigate the structural determinants of the CIAP2:BCL10 interaction, we constructed a series of deletion mutants of cIAP2 (Figure 2A). Co-immunoprecipitation assays revealed that the $\mathrm{NH}_{2}$-terminal BIR region of cIAP2, but not the $\mathrm{COOH}$-terminal CARD domain or the RING domain, mediated its interaction with BCL10 (Figure 2B, lanes 3-6). This finding was confirmed by an in vitro pull-down assay (Figure 2C, lanes 4-9). Previous studies have shown that the BIR region of the IAPs interacts with other IAP targets, including Smac and TRAF2 $(8,10)$. Thus, this region may determine the substrate specificity of the IAPs' E3 activity.

cIAP2 mediates BCL10 ubiquitination and degradation. When cIAP2 and BCL10 were coexpressed in mammalian cells, we detected a series of high-molecular weight BCL10 species characteristic of mono- and polyubiquitinated species at longer exposure times, and this modification was even more pronounced in BCL10 bound to cIAP2 (Figure 3A, lane 4). Western blot analysis using an anti-ubiquitin antibody revealed that BCL10 and/or CIAP2 were ubiquitinated in a manner that was dependent on the cIAP2 RING domain (Figure 3B). To better distinguish cIAP2-mediated BCL10 ubiquitination from cIAP2 self-ubiquitination, we expressed GST-BCL10-FLAG and GST-cIAP2 alone or in combination in $293 \mathrm{~T}$ cells. When GST-cIAP2 was expressed alone and precipitated with glutathione beads, the precipitates contained ubiquitinated products of relatively high molecular weights, corresponding to self-ubiquitination of GSTcIAP2 (Figure 3C, lanes 4 and 8, top panel, range I). However, when GST-BCL10-FLAG was coexpressed with GST-cIAP2, both the antiFLAG and the glutathione precipitates contained ubiquitinated products of high as well as low molecular weights (Figure 3C, lanes 3 and 9, ranges I and II). The low-molecular weight ubiquitinated products were likely due to BCL10 ubiquitination. We further performed an in vitro ubiquitination assay to confirm the cIAP2's E3 activity toward BCL10. BCL10 protein was added to a mixture containing recombinant ubiquitin, ubiquitin-activating enzyme (E1), ubiquitin-conjugating enzyme (E2), and cIAP2 or cIAP2 $\Delta$. In the presence of cIAP2, BCL10 was robustly ubiquitinated (Figure 3D, 

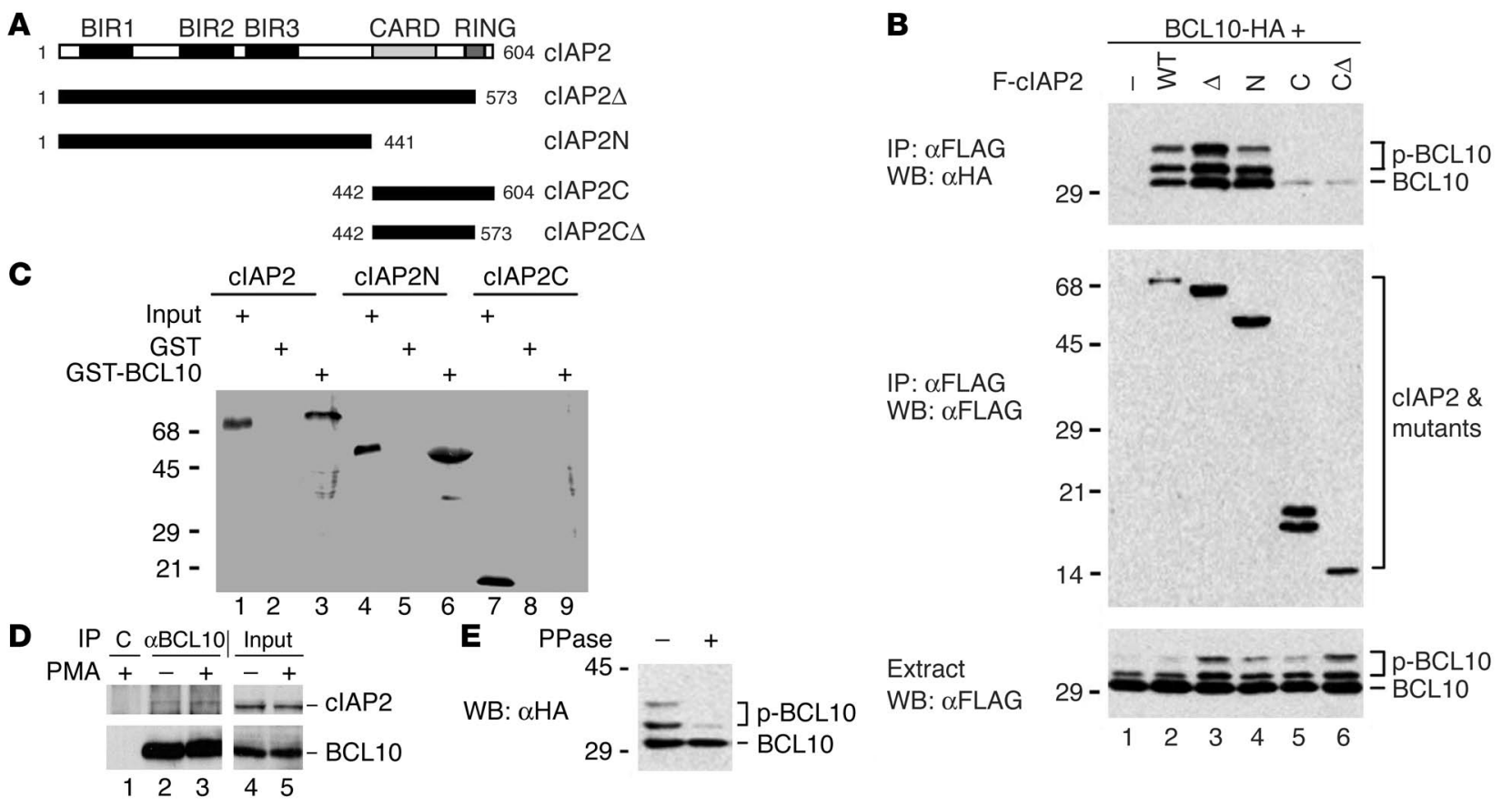

Figure 2

Association of clAP2 and BCL10. (A) Schematic representation of clAP2 and its mutants. The amino acids present in each protein are marked. (B) Interaction of CIAP2 and BCL10 in mammalian cells. 293T cells were transfected with the indicated combinations of plasmids. Cell lysates of the transfected cultures were immunoprecipitated (IP) with an anti-FLAG mAb, and the immunoprecipitated proteins and cell extracts were analyzed by Western blot (WB) with the indicated antibodies. Molecular weight standards (in kDa) are shown on the left. F, FLAG. (C) Interaction of clAP2 and BCL10 in vitro. In vitro-translated, ${ }^{35}$ S-labeled clAP2 proteins were incubated with either GST protein or a GST fusion of BCL10 immobilized on agarose beads. The bound proteins and $5 \%$ of the input ${ }^{35}$ S-clAP2 proteins were analyzed by SDS-PAGE and autoradiography. (D) Association of endogenous BCL10 and clAP2. Extracts from human primary T cells treated with or without PMA plus ionomycin were immunoprecipitated with an anti-BCL10 antibody or an isotype-matching control antibody. The immunoprecipitated proteins and a portion of the input proteins were analyzed by Western blot. (E) Phosphorylation of BCL10 in mammalian cells. Extracts from BCL10-HA-transfected 293T cells were treated with phosphatase (PPase) $(+)$ or left untreated $(-)$.

lane 3). The BCL10 ubiquitination required $\mathrm{E} 1, \mathrm{E} 2$, and the cIAP2 RING domain (Figure 3D, lanes 4-6). Taken together, these results indicate that cIAP2 is a ubiquitin ligase of BCL10.

A recent report suggested that Itch, a HECT-type E3 ligase implicated in antigen receptor signaling, and its close relative, Nedd4, might mediate BCL10 ubiquitination (35). We therefore directly compared the activity of these two E3s with that of cIAP2. Nedd4 and Itch showed very weak ability, if any, to promote BCL10 ubiquitination (Figure 3A, top panel, lanes 2 and 3). Consistently, the interaction of Nedd 4 and Itch with BCL10 was either undetectable or considerably weaker than the cIAP2:BCL10 interaction (Figure 3A). Notably, cIAP2, but not Nedd4 and Itch, also associated with endogenous BCL10 (Figure 3A, lane 4, top panel). Since polyubiquitination often leads to protein degradation, we then tested whether cIAP2 destabilizes BCL10. BCL10 was coexpressed with increasing amounts of CIAP2 or CIAP2A. As the amount of cIAP2 increased, the level of BCL10 decreased (Figure 3E, lanes 1-4). Interestingly, phosphorylated BCL10 was preferentially degraded compared with unphosphorylated BCL10, consistent with the strong binding of phosphorylated BCL10 to cIAP2 (Figure 2B).

cIAP2 inhibits antigen receptor signaling via BCL10 degradation. Given that BCL10 is critical for proliferation and NF- $\kappa B$ activation of lymphocytes in response to antigenic stimulation, we studied the effect of cIAP2 on BCL10 stability and the induction of NF-KB-controlled cytokines in lymphocytes. Notably, in response to antigen receptor simulation in both primary human B and T cells, the level of endogenous BCL10 was significantly reduced within 30 minutes and was not recovered until 16 hours later (Figure 4A). BCL10 plays an analogous role in both B cell receptor and $\mathrm{T}$ cell receptor signaling (21), and we tested BCL10 ubiquitination in T lymphocytes. BCL10 was ubiquitinated in these cells upon treatment with anti-CD3/CD28. In contrast, it was not ubiquitinated or degraded when these cells were treated with the inflammatory cytokine TNF- $\alpha$ (Figure 4B). Thus, ubiquitination and degradation of BCL10 are signal-specific events related to its activation in response to antigen receptor stimulation.

When cIAP2 was introduced into primary human T cells at high transfection efficiency, it significantly destabilized endogenous BCL10 and accelerated anti-CD3/CD28-induced BCL10 degradation (Figure 4C). To address the importance of endogenous cIAP2 in BCL10 degradation, we used the E3-deficient cIAP2, cIAP2 $\Delta$ (Figure 2A). Similar IAP mutants have been shown to exert a dominant-negative effect $(7,10)$. Introduction cIAP2 $\Delta$ into human primary $\mathrm{T}$ cells caused noticeable upregulation of BCL10 $(\sim 70 \%)$ (Figure 4D). cIAP2 $\Delta$ also blocked antigenic stimulationinduced degradation of exogenous BCL10 (Figure 4E), which was normally degraded with kinetics similar to those of endogenous BCL10 (see Supplemental Figure 1; supplemental material available online with this article; doi:10.1172/JCI25641DS1). We next 

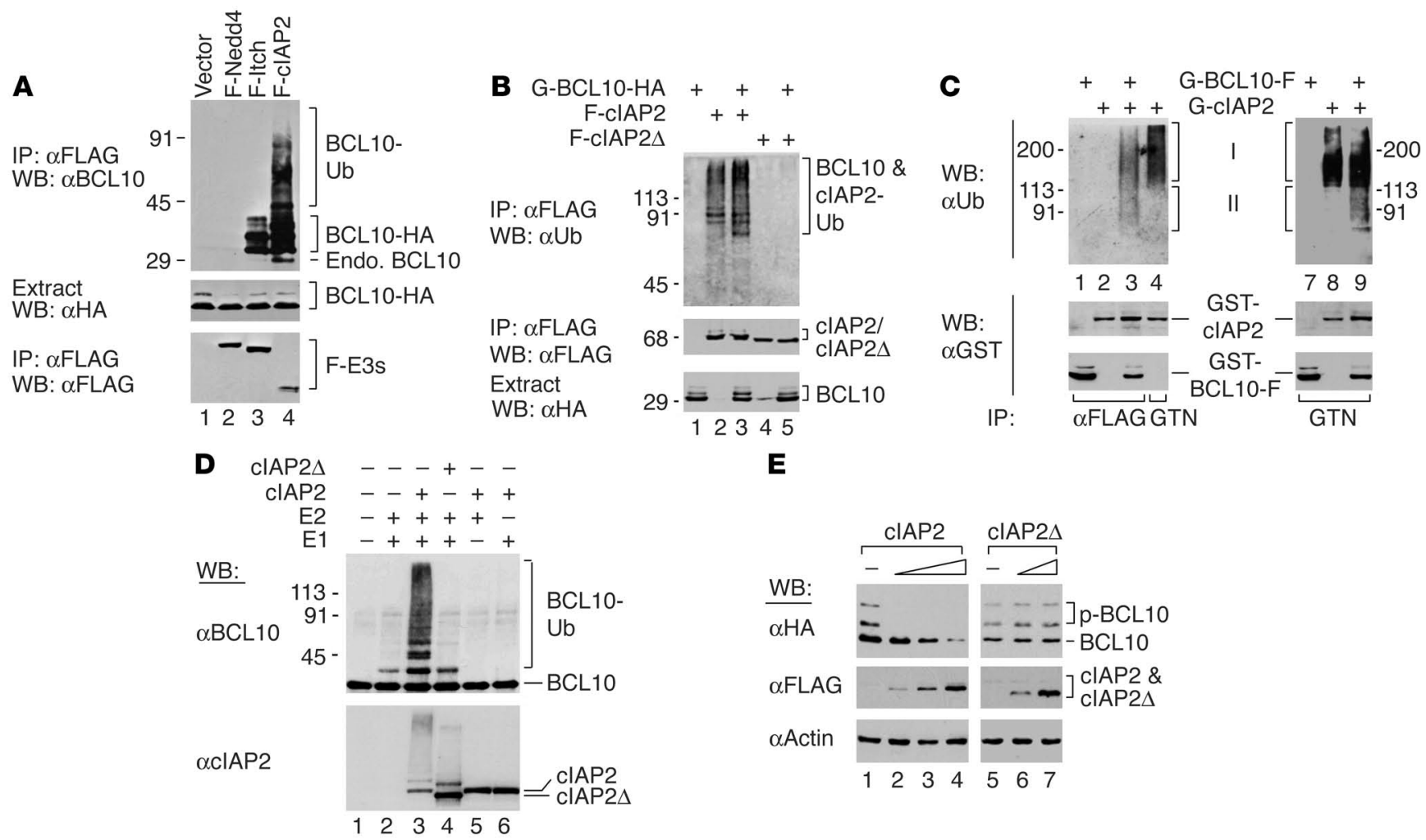

Figure 3

ClAP2 is an E3 of BCL10. (A) ClAP2 mediates BCL10 modification in mammalian cells. BCL10-HA was expressed alone or together with the indicated E3s in the presence of HA-ubiquitin. Varying amounts of BCL10-HA plasmid were used for transfection to ensure similar expression levels among different samples. Anti-FLAG immunoprecipitates and extracts were analyzed by Western blot with the indicated antibodies. This band is recognized by anti-BCL10 antibody and not by anti-HA antibody (data not shown). (B and C) clAP2-mediated BCL10 ubiquitination in cells. (B) 293T cells were transfected with GST-BCL10-HA, FLAG-cIAP2, and FLAG-clAP2 $\triangle$ as indicated, and cell extracts were incubated with anti-FLAG beads. The immunoprecipitates and extracts were analyzed by Western blot. (C) 293T cells were transfected with GST-BCL10-FLAG and/or GST-clAP2. The cell extracts were incubated with either anti-FLAG beads or glutathione beads (GTN, for GST tagged proteins), and the precipitates and extracts were analyzed by Western blot. (D) Ubiquitination of BCL10 by clAP2 in vitro. Recombinant GST-BCL10 was incubated with recombinant ubiquitin, clAP2 (lanes 3, 5, and 6), or clAP2 $\Delta$ (lane 4), and recombinant E1 and/or E2, as indicated. The samples were analyzed by Western blot with anti-BCL10 (top) or anti-clAP2 (bottom) antibody. clAP2 did not ubiquitinate GST (data not shown). (E) Wild-type clAP2, but not the RING deletion mutant, induces BCL10 degradation. BCL10-HA was coexpressed with increasing amounts of FLAG-ClAP2 or FLAG-cIAP2 $\triangle$. Endo., endogenous; F, FLAG; G, GST; Ub, ubiquitin.

determined the effect of cIAP2 and cIAP2 $\Delta$ on anti-CD3/CD28mediated induction of IL-2, a key mediator of $\mathrm{T}$ cell expansion whose expression is dependent on BCL10 and NF-kB activation. Because IL-2 expression in human $\mathrm{T}$ cells also strictly requires CD28 costimulation, to induce IL-2 expression only in transfected cells, we used a chimeric construct consisting of the murine CD28 extracellular domain and human CD28 cytoplasmic tail (m/hCD28), which, when stimulated with agonistic anti-murine CD28 antibody, provides appropriate costimulatory signal for IL-2 induction in human T cells (36). We introduced cIAP2, IAP $2 \Delta$, or vector into primary human $\mathrm{T}$ lymphocytes together with $\mathrm{m} / \mathrm{hCD} 28$. When the vector-transfected cell cultures were treated with beads coated with anti-human CD3 and antimurine CD28, IL-2 expression was highly induced (Figure 4F, column 2 versus 1). Interestingly, cIAP2 strongly inhibited IL-2 production (over $60 \%$ ), while cIAP $2 \Delta$ markedly enhanced it $(\sim 300 \%)$ (Figure 4F, columns 3 and 4$)$. Together, these results suggest that cIAP2 plays an important role in downregulating antigen receptor signaling via BCL10 degradation.
Loss of E3 activity of cIAP2 in t(11;18) MALT lymphomas. All of the cIAP2-MALT1 fusion proteins found in $\mathrm{t}(11 ; 18)$ lymphomas lack an intact cIAP2 RING domain $(18,30)$. To confirm that these fusion proteins lose their E3 activity, we constructed a cIAP2-MALT1 protein consisting of the first 441 amino acids of cIAP2 and the paracaspase domain of MALT1, which represents the majority of clinically observed cIAP2-MALT1 fusions (18, 30) (Figure 5A). As expected, cIAP2-MALT1 was much more stable than cIAP2 when introduced into mammalian cells (Figure 5A), and it retained the ability to bind to BCL10 as judged by co-immunoprecipitation assay (Figure 5B, lane 3 ), in agreement with the CIAP2 BIR region being responsible for the interaction (Figure 2, B and C). However, cIAP2-MALT1 failed to ubiquitinate BCL10 (Figure 5B, lane 3). Like cIAP2 $\Delta$, cIAP2MALT1 impeded BCL10 degradation in human primary $\mathrm{T}$ cells (Figure 4D, lane 3, and Figure 5C, lanes $4-6$ versus $1-3$ ) and enhanced anti-CD3/CD28-induced IL-2 production (Figure 4F, column 5). Therefore, the $t(11 ; 18)$ translocation results in a fusion protein that not only lacks the E3 ubiquitin ligase activity of cIAP2 but also may interfere with the function of endogenous cIAP2. 
A

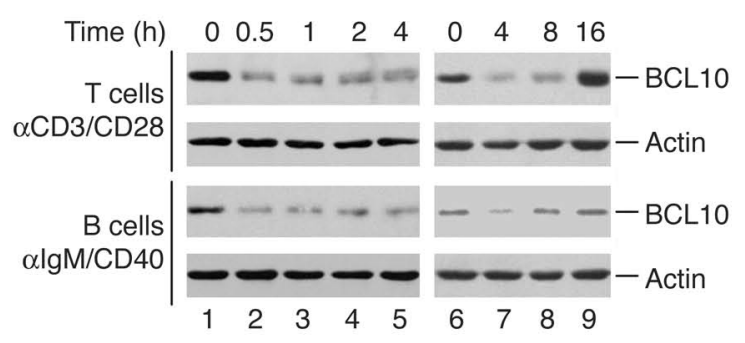

B $\alpha$ CD3/CD28

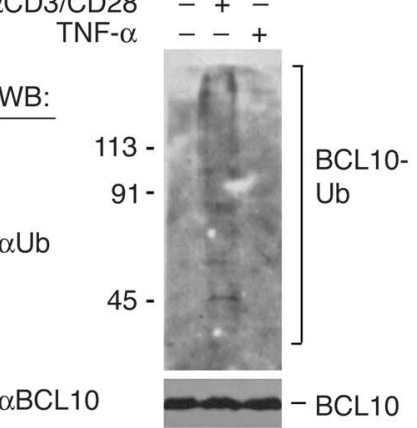

C

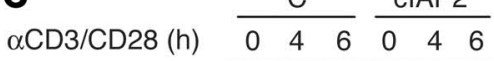
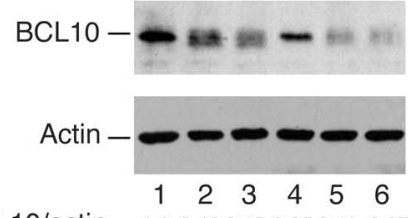

$\begin{array}{lllllllll} & \text { BCL10/actin } \quad 1.0 & 0.46 & 0.19 & 0.35 & 0.11 & 0.07\end{array}$

$\mathbf{F}$

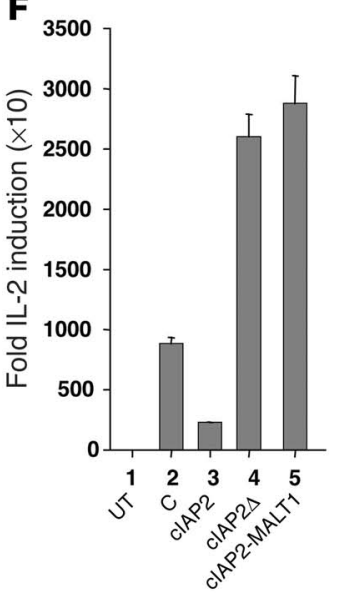

Figure 4

ClAP2 inhibits antigen receptor signaling via BCL10 degradation. (A) Degradation of BCL10 in response to antigenic stimulation. Human primary $T$ and $B$ cells were treated with anti-CD3/CD28 and anti-IgM/CD40, respectively, for the indicated durations. (B) Ubiquitination of BCL10 after antigen receptor cross-linking in primary T lymphocytes. Human primary T cells were treated with anti-CD3/CD28 and TNF- $\alpha$ as indicated. BCL10 was immunoprecipitated from cell lysates and analyzed by Western blot. (C) CIAP2 accelerates the degradation of endogenous BCL10. Human $\mathrm{CD}^{+}$cells were transfected with a control GFP-expressing plasmid (C) or clAP2 plasmid. Cells were then treated with anti-CD3/CD28 beads for the indicated durations. The transfection efficiency was approximately $80 \%$ based on GFP expression. (D) cIAP2 $\Delta$ and cIAP2-MALT1 stabilize BCL10. Human primary CD4+ T cells were transfected with GFP (C), cIAP2A, or cIAP2-MALT1, plus murine CD8. Transfected live cells were purified using anti-murine $C D 8$ beads. (E) Effects of CIAP2 $\Delta$ on the degradation of BCL10. Human primary CD4+ $T$ cells were transfected with HA-BCL10 together with GFP (C) or clAP2A. Cells were then treated with anti-CD3/CD28 for the indicated durations, and the levels of HA-BCL10 were analyzed by Western blot with anti-HA antibody. (F) CIAP2 inhibits anti-CD3/CD28-mediated IL-2 induction. Human primary $\mathrm{CD}^{+} \mathrm{T}$ cells were transfected with GFP, cIAP2, cIAP2A, or clAP2-MALT1, plus m/hCD28. Cells were stimulated with anti-human CD3 and anti-murine CD28 beads for 8 hours or left untreated. The fold IL-2 induction relative to untreated cells (UT) is shown. The data are representative of 3 independent experiments.

The upregulation of BCL10 in $\mathrm{t}(11 ; 18) /$ cIAP2-MALT1 lymphomas and the ability of CIAP2-MALT1 to interact with BCL10 led us to test whether BCL10 participates in CIAP2-MALT1-induced NF-кB activation. Because high levels of ectopic expression of cIAP2MALT1 lead to robust NF- $\mathrm{KB}$ activation, we transfected Jurkat $T$ cells with varying amounts of BCL10 together with a relatively small amount of cIAP2-MALT1, which should more closely resemble its typical level in $\mathrm{t}(11 ; 18)$ MALT lymphomas as opposed to high levels of overexpression. Notably, under these conditions, BCL10 and cIAP2-MALT1 activated NF- $\kappa$ B synergistically (Figure 5D). We also examined the effect of BCL10 downregulation on cIAP2MALT1-induced NF-кB activation. BCL10 is expressed in various cell lines (Supplemental Figure 2). The expression of endogenous BCL10 could be effectively knocked down in HeLa cells using the RNA interference technique (Figure 5E). Expression of cIAP2MALT1 in HeLa cells activated an NF-אB-responsive reporter gene, and, interestingly, this activation was attenuated when the level of
BCL10 was lowered by small interfering RNA (siRNA) (Figure 5E). Therefore, cIAP2-MALT1 not only acts in synergy with but also at

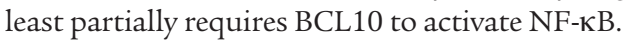

\section{Discussion}

The association of MALT lymphoma with 3 independent translocations is unique among small B cell lymphomas, where typically only 1 translocation is involved, as is evident in Burkitt lymphoma $(c-m y c)$, follicular lymphoma (bcl2), and mantle cell lymphoma (bcl1/cyclin D1) (37). Previous studies have revealed a critical role of both BCL10 and MALT1 in lymphocyte proliferation and NF-кB activation. The current study indicates that cIAP2 normally mediates BCL10 degradation and inhibits antigen receptor signaling, and that this function is missing in the cIAP2-MALT1 fusion, likely leading to enhanced BCL10 stability in $\mathrm{t}(11 ; 18)$ lymphomas. Therefore, remarkably, all 3 proteins involved in MALT lymphomas are functionally linked in antigenic signaling to NF- $\mathrm{KB}$. In addition, high levels of BCL10 pro- 
A
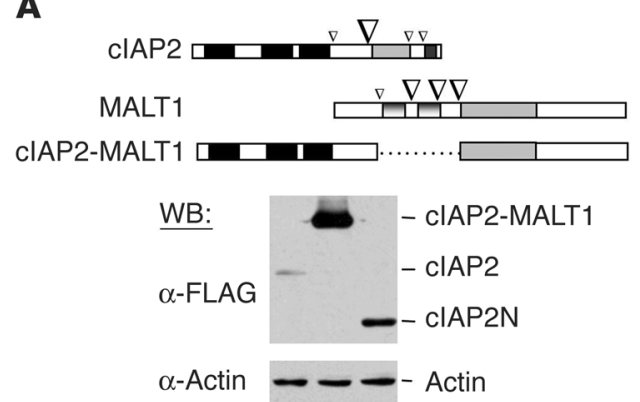

D

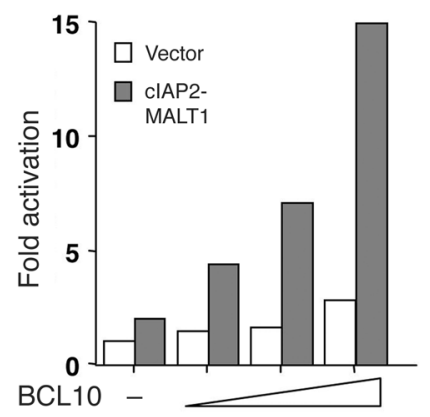

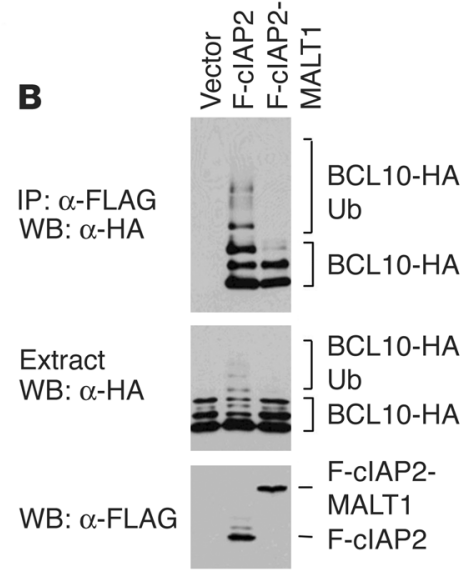

E

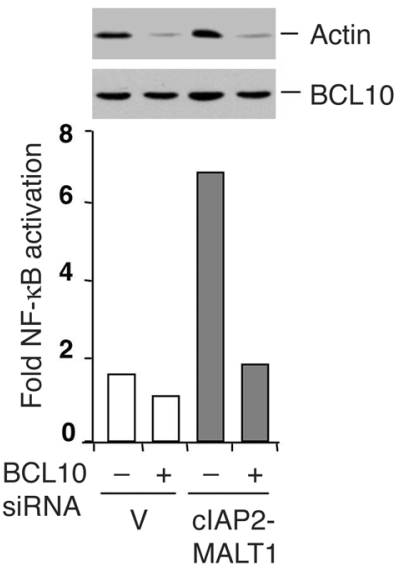

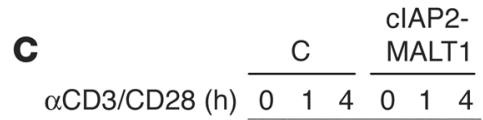

$\mathrm{HA}-\mathrm{BCL} 10-\ldots=$

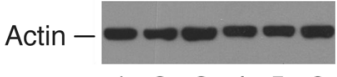

HA-BCL10/actin $\quad 10.2001 .910 .1$
$\mathbf{F}$

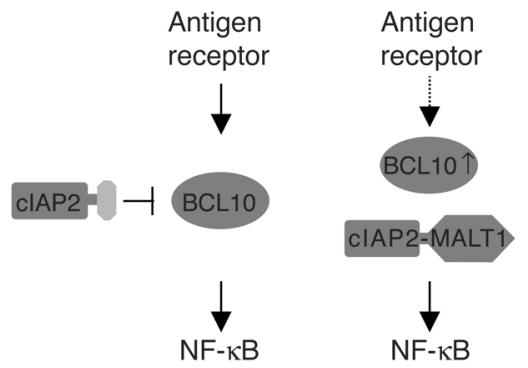

\section{Figure 5}

BCL10 is not ubiquitinated by the cIAP2-MALT1 fusion protein and is involved in clAP2-MALT1 signaling to NF-KB. (A) Schematic diagram of cIAP2-MALT1. The major and minor breakage points found in $\mathrm{t}(11 ; 18)$ lymphomas are indicated by large and small arrowheads, respectively. Bottom: 293T cells were transfected with equal amounts of the indicated plasmids. (B) The clAP2-MALT1 fusion cannot ubiquitinate BCL10. BCL10-HA was expressed with either FLAG-cIAP2 or FLAG-clAP2-MALT1. Anti-FLAG immunoprecipitates and extracts were analyzed by Western blotting. (C) clAP2-MALT1 fusion inhibits BCL10 degradation. Primary human T cells were transfected with GFP (C) or clAP2-MALT1 and treated as in Figure 4D. (D) BCL10 and clAP2-MALT1 activate NF-KB synergistically. Increasing amounts of BCL10 were transfected into

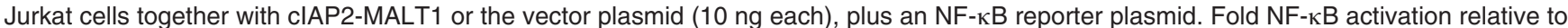
the vector-transfected control is shown. (E) Knocking down of BCL10 expression by siRNA decreases clAP2-MALT1-induced NF- $\kappa B$ activation. HeLa cells treated with BCL10 siRNA or a control siRNA were transfected with the vector or clAP2-MALT1, plus an NF-kB-responsive reporter plasmid. Relative levels of NF-KB activity and the expression of BCL10 are shown. V, vector. $(F)$ A model for the role of clAP2 in antigen receptor signaling and its dysregulation in $\mathrm{t}(11 ; 18)$ lymphomas. Left: In normal lymphocytes, clAP2 mediates BCL10 ubiquitination and degradation in response to antigen receptor stimulation. Right: In t $(11 ; 18)$ MALT lymphomas, the E3 activity of clAP2 is missing, resulting in enhanced stability of BCL10, which acts together with clAP2-MALT1 to activate NF-KB independently of antigen receptor stimulation.

tein are also associated with $\mathrm{t}(14 ; 18) / \mathrm{I} g H-M A L T 1$-positive MALT lymphomas and are believed to be critical for MALT1-mediated NF-KB activation in these tumor cells (32). Thus, BCL10 upregulation appears to be a unifying molecular mechanism of MALT lymphomas associated with all 3 distinct translocations. The strength and duration of antigenic signaling are central to proper regulation of immune responses, and E3 ubiquitin ligases have recently emerged as important regulators of lymphocyte activation (38). The rapid and robust degradation of BCL10 in both primary human B and $\mathrm{T}$ lymphocytes, in combination with its essential role in lymphocyte proliferation, indicates that the level of BCL10 may be a focal point for the regulation of antigen receptor signaling. Through the control of BCL10 stability, cIAP2 likely plays a critical role in downregulating lymphocyte activation and preventing hyperproliferation.
Finally, the results in this and previous reports also indicate that cIAP2-MALT1 is a unique oncoprotein: it is both a gain-of-function (for MALT1) and a loss-of-function (for cIAP2) mutant. When expressed in primary $\mathrm{T}$ cells, both cIAP2 and cIAP2-MALT1 enhance IL-2 production to similar levels, suggesting the importance of the loss of function in lymphocyte activation. The relative contribution of these 2 properties to the oncogenic potential of cIAP2-MALT1 has yet to be determined. It is of note that while cIAP2-MALT1 is able to strongly activate NF- $\mathrm{KB}$ upon overexpression, it is not highly expressed in $\mathrm{t}(11 ; 18)$-positive MALT lymphomas $(29,32)$. Nevertheless, the combination of both properties of cIAP2-MALT1 likely results in strong NF-kB activation in lymphocytes harboring cIAP2-MALT1 in the presence of weak or no antigenic stimulation (Figure 5F), eventually leading to oncogenic transformation. 


\section{Methods}

Antibodies and plasmids. Antibodies against CD40 (BD Biosciences Pharmingen), IgM (MP Biochemicals), HA tag, BCL10, ubiquitin, cIAP2 (Santa Cruz Biotechnology Inc.), and FLAG tag (Sigma-Aldrich) were purchased from the indicated sources. Beads coated with anti-human CD3 and anti-human CD28, with anti-human CD3 and anti-murine CD28, or with anti-murine CD28 only were prepared as previously described (36). All constructs for expressing proteins in mammalian cells and for in vitro transcription/translation were made in pRK5 with tags at the $\mathrm{NH}_{2}$ - and/or C-terminus as indicated. Constructs for expressing GST-BCL10, -BCL10N, and -BCL10C fusion proteins in E. coli were made in pGEX-1ZT.

Transfection, immunoprecipitation, Western blotting, and phosphatase treatment. Transient transfection of $293 \mathrm{~T}$ cells was performed using the calcium phosphate precipitation method, and $0.01-4 \mu \mathrm{g}$ of each plasmid was used to achieve similar levels of protein expression unless otherwise indicated. Sixteen to 22 hours after transfection, cell lysates were prepared in 1\% NP-40 lysis buffer (50 mM HEPES, $150 \mathrm{mM} \mathrm{NaCl,} \mathrm{10 \%} \mathrm{glycerol,} 2 \mathrm{mM}$ DTT, 1\% NP-40, and $1 \mathrm{mM}$ EDTA) and immunoprecipitated with anti-FLAG $\mathrm{mAb}$ M2 immobilized on beads. The immunoprecipitates and cell lysates were resolved by SDS-PAGE and analyzed by Western blot with the indicated antibodies. BCL10 immunohistochemistry of MALT lymphomas was carried out as previously described $(33,39)$. For Western blotting, all the tumors were previously characterized and at advanced clinical stages. Each sample contained a similar portion (more than 70\%) of tumor cells as judged by histological examination of the H\&E staining. To detect endogenous BCL10: cIAP2 interaction, freshly isolated human primary $\mathrm{CD}^{+} \mathrm{T}$ cells were first expanded by stimulation with anti-CD3/CD28 beads (3 beads per cell) and culturing for approximately 12 days (36). Cells were then either stimulated with $10 \mathrm{ng} / \mathrm{ml}$ PMA plus $100 \mathrm{ng} / \mathrm{ml}$ ionomycin (Sigma-Aldrich) or left untreated. Cell lysates from approximately $2.5 \times 10^{7}$ cells were subject to a co-immunoprecipitation assay.

In vitro binding and ubiquitination assays. Recombinant GST, GST-BCL10, GST-cIAP2, GST-cIAP2 $\Delta$, His ${ }_{6}$-tagged yeast E1 Uba1, E2 UbcH5a, and ubiquitin were expressed in E. coli DH5 $\alpha$ or BL21 and purified with glutathione or $\mathrm{Ni}^{2+}$ beads (QIAGEN) as previously described (40). For in vitro binding, full-length cIAP2 protein and its truncation mutants were generated by the TNT coupled in vitro transcription/translation system (Promega) according to the manufacturer's instructions and incubated with GST or GST-BCL10 in $0.5 \mathrm{ml}$ of NP-40 lysis buffer at $4^{\circ} \mathrm{C}$ for 2 hours. The immunoprecipitates were resolved by SDS-PAGE and analyzed by autoradiography. For in vitro ubiquitination assay, GST-BCL10 was added to a mix of E1 Uba1 (50 ng), E2 UbcH5a (200 ng), ubiquitin (0.8 $\mu \mathrm{g})$, and in vitro-translated GST-cIAP2 or GST-cIAP2 $\Delta$ in a total volume of $30 \mu \mathrm{l}$ of ubiquitination buffer (50 mM Tris, pH 7.5, 2 mM ATP, $2.5 \mathrm{mM} \mathrm{MgCl}_{2}, 0.5 \mathrm{mM}$ DTT, and $0.05 \% \mathrm{NP}-40$ ). The mix was incubated at $30^{\circ} \mathrm{C}$ for 1 hour. The reactions were terminated with sample buffer and resolved on SDS-PAGE.

$N F-\kappa B$ reporter assay. Jurkat and HeLa cells were transfected with the indicated potential NF- $\kappa \mathrm{B}$ activators, $\mathrm{P}(\kappa \mathrm{x})_{3}$-IFN-LUC (NF- $\mathrm{KB}$-responsive luciferase), and pcDNA3-LacZ by Lipofectamine 2000 (Invitrogen Corp.) and SuperFect (QIAGEN) methods, respectively. The total amount of plasmid DNA was kept constant by addition of empty vector. After culturing for 24 hours, cell lysates were made, and luciferase activity was determined by a luciferase assay system (Promega) according to the manufacturer's instructions. The luciferase activity was normalized with the expression of $\beta$-galactosidase, which was measured using $o$-nitrophenyl- $\beta$-D-galactopyranoside.

Analysis of BCL10 degradation and modification and IL-2 production in buman primary $T$ cells. Human peripheral blood lymphocytes were isolated from normal volunteer donors after apheresis and elutriation, and B, total $\mathrm{T}$, and $\mathrm{CD}^{+} \mathrm{T}$ cells were purified by negative selection using magnetic beads (Dynal Biotech) as previously described (36). Expression plasmids were introduced into the $\mathrm{CD}^{+} \mathrm{T}$ cells using an Amaxa electoporator (Amaxa Inc.) according to the manufacturer's instructions. Typically, $5 \times 10^{6}$ cells and $0.4-1.2 \mu \mathrm{g}$ of each plasmid were used, and the total amounts of DNA were kept constant by addition of the vector DNA. Transfection efficiency was approximately $20-80 \%$ and was consistent within each experiment as judged by GFP expression and/or FACS analysis. Fourteen to 20 hours after transfection, cells were stimulated with beads coated with anti-human CD3 and anti-human CD28 or with anti-human CD3 and anti-murine CD28 (3 beads per cell) as previously described (36). To purify transfected cells, a plasmid expressing the murine CD8 extracellular domain was cotransfected into primary human $T$ cells. The apoptotic cells were removed using a Dead Cell Removal Kit (Miltenyi Biotec), and the transfected live cells were purified using anti-CD8 microbeads (Miltenyi Biotec). Over $98 \%$ of purified cells were positive for murine CD8 as judged by FACS analysis. To examine IL-2 induction, cytoplasmic RNA was purified by RNeasy Kits (QIAGEN) and reverse transcribed using First-strand cDNA Synthesis System for Quantitative RT-PCR (Marligen Biosciences). Primers and probes to detect IL-2 and 28S rRNA were designed using Primer Express software (Applied Biosytems). Their sequences are: CACCAGGATGCTCACATTTAAGTT and GCTAAATTCAGCACTTCCTCCAG (IL-2 primers), CATGCCCAAGAAGGCCACAGAACTG (IL-2 probe), GAACGTACCATCTGTGGGATTATGA and CTATCCGAGGCCAACCGA (28S primers), and CCTCTAAGTCAGAATCCCGCCCAGGC (28S probe). Realtime PCR amplification and product detection were performed using the ABI PRISM 7700 (Applied Biosytems) as recommended by the manufacturer. Results were normalized to $28 \mathrm{~S}$ rRNA levels, and relative expression was determined using the $\Delta \Delta \mathrm{Ct}$ method according to the manufacturer's protocol. The $\mathrm{m} / \mathrm{hCD} 28$-cotransfected cells were purified using anti-murine CD28 beads. To examine BCL10 ubiquitination, expanded CD4 ${ }^{+}$T cells (see above) were treated with the indicated reagents. Cell extracts were prepared from approximately $1 \times 10^{8}$ untreated and TNF- $\alpha$-treated cells and approximately $3 \times 10^{8}$ anti-CD3/CD28-treated cells to normalize the level of BCL10, and in lysis buffer containing 1\% SDS to disrupt protein-protein interactions. The lysates were then diluted in buffer with no SDS and incubated with a polyclonal anti-BCL10 antibody that was conjugated to AminoLink Plus Coupling Gel (Pierce Biotechnology Inc.).

Statistics. Pooled $t$ test was used to evaluate the relative level of BCL10 (BCL10/actin) from the $\mathrm{t}(11 ; 18)$-positive versus control groups of MALT lymphomas without any known translocation. The computed $P$ value $(P=0.0176)$ was significantly lower than traditionally set criterion $(P<0.05)$.

\section{Acknowledgments}

We thank Rachael Felberbaum, Natalie Howe, and Stephen Stolzfus for excellent technical assistance; R. Felberbaum and N. Howe for scientific editing; Jun Tang and Mike Milone for helpful discussion; and M. Celeste Simon for the Amaxa electroporator. We also thank the Human Immunology Core of the University of Pennsylvania's Center for AIDS Research for providing human primary lymphocytes. This work was supported by NIH grants CA88868 and GM60911 (to X. Yang); NIH grant AI057838 (to J.L. Riley); the Leukemia Research Fund, United Kingdom (to M.-Q. Du); and Fonds voor Wetenschappelijk Onderzoek-Vlaanderen (to P. Marynen). X. Yang is a Scholar of the Leukemia \& Lymphoma Society.

Received for publication May 13, 2005, and accepted in revised form October 4, 2005.

Address correspondence to: Xiaolu Yang, 421 Curie Boulevard, Room 610, Philadelphia, Pennsylvania 19104-6160, USA. Phone: (215) 5736739; Fax: (215) 573-6725; E-mail: xyang@mail.med.upenn.edu. 
1. Deveraux, Q.L., and Reed, J.C. 1999. IAP family proteins: suppressors of apoptosis. Genes Dev. 13:239-252.

2. Salvesen, G.S., and Duckett, C.S. 2002. IAP proteins: blocking the road to death's door. Nat. Rev. Mol. Cell Biol. 3:401-410.

3. Deveraux, Q.L., et al. 1998. IAPs block apoptotic events induced by caspase- 8 and cytochrome $c$ by direct inhibition of distinct caspases. EMBO J. 17:2215-2223.

4. Du, C., Fang, M., Li, Y., Li, L., and Wang, X. 2000. Smac, a mitochondrial protein that promotes cytochrome c-dependent caspase activation by eliminating IAP inhibition. Cell. 102:33-42.

5. Verhagen, A.M., et al. 2000. Identification of DIABLO, a mammalian protein that promotes apoptosis by binding to and antagonizing IAP proteins. Cell. 102:43-53.

6. Srinivasula, S.M., et al. 2001. A conserved XIAPinteraction motif in caspase- 9 and Smac/DIABLO regulates caspase activity and apoptosis. Nature. 410:112-116.

7. Yang, Y., Fang, S., Jensen, J.P., Weissman, A.M., and Ashwell, J.D. 2000. Ubiquitin protein ligase activity of IAPs and their degradation in proteasomes in response to apoptotic stimuli. Science. 288:874-877.

8. Hu, S., and Yang, X. 2003. Cellular inhibitor of apoptosis 1 and 2 are ubiquitin ligases for the apoptosis inducer Smac/DIABLO. J. Biol. Chem. 278:10055-10060.

9. Vaux, D.L., and Silke, J. 2005. IAPs, RINGs and ubiquitylation. Nat. Rev. Mol. Cell Biol. 6:287-297.

10. Li, X., Yang, Y., and Ashwell, J.D. 2002. TNF-RII and c-IAP1 mediate ubiquitination and degradation of Traf2. Nature. 416:345-349.

11. Isaacson, P.G., and Du, M.Q. 2004. MALT lymphoma: from morphology to molecules. Nat. Rev. Cancer. 4:644-653.

12. Cavalli, F., Isaacson, P.G., Gascoyne, R.D., and Zucca, E. 2001. MALT lymphomas. Hematology (Am. Soc. Hematol. Educ. Program). 2001:241-258.

13. Zhang, Q., et al. 1999. Inactivating mutations and overexpression of BCL10, a caspase recruitment domain-containing gene, in MALT lymphoma with $\mathrm{t}(1 ; 14)(\mathrm{p} 22 ; \mathrm{q} 32)$. Nat. Genet. 22:63-68.

14. Willis, T.G., et al. 1999. Bcl10 is involved in $\mathrm{t}(1 ; 14)(\mathrm{p} 22 ; \mathrm{q} 32)$ of MALT B cell lymphoma and mutated in multiple tumor types. Cell. 96:35-45.

15. Sanchez-Izquierdo, D., et al. 2003. MALT1 is deregulated by both chromosomal translocation and amplification in B-cell non-Hodgkin lymphoma. Blood. 101:4539-4546.

16. Streubel, B., et al. 2003. T(14;18)(q32;q21) involving IGH and MALT1 is a frequent chromosomal aberration in MALT lymphoma. Blood. 101:2335-2339.

17. Uren, A.G., et al. 2000. Identification of paracaspases and metacaspases: two ancient families of caspase-like proteins, one of which plays a key role in MALT lymphoma. Mol. Cell. 6:961-967.

18. Dierlamm, J., et al. 1999. The apoptosis inhibitor gene API2 and a novel 18q gene, MLT, are recurrently rearranged in the $\mathrm{t}(11 ; 18)(\mathrm{q} 21 ; \mathrm{q} 21)$ associated with mucosa-associated lymphoid tissue lymphomas. Blood. 93:3601-3609.

19. Akagi, T., et al. 1999. A novel gene, MALT1 at $18 \mathrm{q} 21$, is involved in $\mathrm{t}(11 ; 18)(\mathrm{q} 21 ; \mathrm{q} 21)$ found in low-grade B-cell lymphoma of mucosa-associated lymphoid tissue. Oncogene. 18:5785-5794.

20. Morgan, J.A., et al. 1999. Breakpoints of the $\mathrm{t}(11 ; 18)(\mathrm{q} 21 ; \mathrm{q} 21)$ in mucosa-associated lymphoid tissue (MALT) lymphoma lie within or near the previously undescribed gene MALT1 in chromosome 18. Cancer Res. 59:6205-6213.

21. Ruland, J., et al. 2001. Bcl10 is a positive regulator of antigen receptor-induced activation of NF-kap$\mathrm{paB}$ and neural tube closure. Cell. 104:33-42.

22. Xue, L., et al. 2003. Defective development and function of Bcl10-deficient follicular, marginal zone and B1 B cells. Nat. Immunol. 4:857-865.

23. Ruland, J., Duncan, G.S., Wakeham, A., and Mak, T.W. 2003. Differential requirement for Malt1 in $\mathrm{T}$ and $\mathrm{B}$ cell antigen receptor signaling. Immunity. 19:749-758.

24. Ruefli-Brasse, A.A., French, D.M., and Dixit, V.M. 2003. Regulation of NF-kappaB-dependent lymphocyte activation and development by paracaspase. Science. 302:1581-1584.

25. Lucas, P.C., et al. 2001. Bcl10 and MALT1, independent targets of chromosomal translocation in malt lymphoma, cooperate in a novel NF-kappa B signaling pathway. J. Biol. Chem. 276:19012-19019.

26. Zhou, H., et al. 2004. Bcl10 activates the NF-kap$\mathrm{paB}$ pathway through ubiquitination of NEMO. Nature. 427:167-171.

27. Sun, L., Deng, L., Ea, C.K., Xia, Z.P., and Chen, Z.J. 2004. The TRAF6 ubiquitin ligase and TAK1 kinase mediate IKK activation by BCL10 and MALT1 in T lymphocytes. Mol. Cell. 14:289-301.

28. Hayden, M.S., and Ghosh, S. 2004. Signaling to NF-kappaB. Genes Dev. 18:2195-2224.

29. Zhou, H., Du, M.Q., and Dixit, V.M. 2005. Constitu- tive NF-kappaB activation by the $\mathrm{t}(11 ; 18)(\mathrm{q} 21 ; \mathrm{q} 21)$ product in MALT lymphoma is linked to deregulated ubiquitin ligase activity. Cancer Cell. 7:425-431.

30. Ye, H., et al. 2003. Variable frequencies of $\mathrm{t}(11 ; 18)(\mathrm{q} 21 ; \mathrm{q} 21)$ in MALT lymphomas of different sites: significant association with CagA strains of $\mathrm{H}$ pylori in gastric MALT lymphoma. Blood. 102:1012-1018.

31. Maes, B., Demunter, A., Peeters, B., and De WolfPeeters, C. 2002. BCL10 mutation does not represent an important pathogenic mechanism in gastric MALT-type lymphoma, and the presence of the API2-MLT fusion is associated with aberrant nuclear BCL10 expression. Blood. 99:1398-1404.

32. Ye, H., et al. 2005. MALT lymphoma with $\mathrm{t}(14 ; 18)(\mathrm{q} 32 ; \mathrm{q} 21) / \mathrm{IGH}-\mathrm{MALT} 1$ is characterized by strong cytoplasmic MALT1 and BCL10 expression. J. Pathol. 205:293-301.

33. Ye, H., et al. 2000. BCL10 expression in normal and neoplastic lymphoid tissue. Nuclear localization in MALT lymphoma. Am. J. Pathol. 157:1147-1154.

34. Thome, M., et al. 2001. Equine herpesvirus protein E10 induces membrane recruitment and phosphorylation of its cellular homologue, bcl-10. J. Cell Biol. 152:1115-1122.

35. Scharschmidt, E., Wegener, E., Heissmeyer, V., Rao, A., and Krappmann, D. 2004. Degradation of Bcl10 induced by T-cell activation negatively regulates NFkappa B signaling. Mol. Cell. Biol. 24:3860-3873.

36. Parry, R.V., Rumbley, C.A., Vandenberghe, L.H., June, C.H., and Riley, J.L. 2003. CD28 and inducible costimulatory protein Src homology 2 binding domains show distinct regulation of phosphatidylinositol 3-kinase, Bcl-xL, and IL-2 expression in primary human CD4 T lymphocytes. J. Immunol. 171:166-174.

37. Willis, T.G., and Dyer, M.J. 2000. The role of immunoglobulin translocations in the pathogenesis of B-cell malignancies. Blood. 96:808-822.

38. Duan, L., Reddi, A.L., Ghosh, A., Dimri, M., and Band, H. 2004. The Cbl family and other ubiquitin ligases: destructive forces in control of antigen receptor signaling. Immunity. 21:7-17.

39. Liu, H., et al. 2001. T(11;18)(q21;q21) is associated with advanced mucosa-associated lymphoid tissue lymphoma that expresses nuclear BCL10. Blood. 98:1182-1187.

40. Park, S.M., Yoon, J.B., and Lee, T.H. 2004. Receptor interacting protein is ubiquitinated by cellular inhibitor of apoptosis proteins (c-IAP1 and c-IAP2) in vitro. FEBS Lett. 566:151-156. 